\title{
Cálculo referencial de material particulado en el aire como factor de contaminación ambiental en el área urbana de la ciudad de Pujilí
}

\section{(Referential calculation of particulate matter in the air as a factor of environmental pollution in the urban area of the city of Pujilí)}

\author{
Paola Vallejo Choez ${ }^{1}$, Carmen González Moya ${ }^{1}$, Fredy Mena Mora ${ }^{2}$
}

\begin{abstract}
Resumen:
El presente estudio constituye una investigación preliminar sobre la calidad ambiental de la ciudad de Pujilí, realizado a partir de la recolección de muestras de material particulado y el conteo de tránsito vehicular en seis puntos de la ciudad. El proceso metodológico se fundamenta en lo establecido en el Texto Unificado de la Legislación Ambiental Secundaria para la medición de material particulado atmosférico, y la utilización de tablas de conteo para el registro de tránsito vehicular. Los resultados obtenidos reflejan la incidencia del tránsito vehicular, las características de la capa de rodadura vial, la erosión del suelo, y el clima en la contaminación del aire y su repercusión en la salud de la población.
\end{abstract}

Palabras clave: calidad ambiental, contaminación atmosférica; polución; deterioro del entorno; salud pública

\begin{abstract}
:
This is a preliminary investigation on the environmental quality of the city of Pujilí, made from the collection of samples of particulate matter and vehicular traffic counts on six points of the city. The methodology is based on the provisions of the Unified Text of Secondary Environmental Legislation for measuring atmospheric particulate matter, and the use of count tables for vehicle registration. The results reflect the impact of vehicular traffic, the characteristics of the rolling road layer, soil erosion, and climate on air pollution and its impact on the health of the population.
\end{abstract}

Keywords: environmental quality; air pollution; pollution; deterioration of the environment; public health.

\footnotetext{
${ }^{1}$ Universidad Tecnológica Equinoccial, Quito - Ecuador ( \{paola.vallejo,carmen.gonzalez\} @ute.edu.ec )

${ }^{2}$ Universidad Central del Ecuador (frmena@uce.edu.ec)
} 


\section{Introducción}

El efecto de la contaminación del aire exterior es mayor en zonas urbanas, provoca deterioro en la salud de las poblaciones y es causa de problemas respiratorios (Organización Panamericana de la Salud, 2005). En el Ecuador las ciudades grandes como Quito muestran niveles mayores de contaminación, pero el estudio evidencia que en ciudades intermedias tipo B (GADP de Cotopaxi, 2015) como Pujilí, la problemática también existe y se incrementa.

La contaminación del aire en la zona de estudio se produce por diversas actividades del ser humano (factores antrópicos) y por causas naturales como aquellas que provienen del proceso eruptivo del volcán Cotopaxi, como influencia directa, y del volcán Tungurahua como influencia indirecta, así como de la erosión del suelo (Organización Panamericana de la Salud, 2005). Las causas que dan origen a la contaminación antrópica en la zona de estudio son diversas, originadas por las actividades industriales, comerciales, domésticas, agropecuarias y de transporte.

La contaminación causada por el transporte es compleja; en Ecuador el combustible utilizado en los distintos tipos de vehículos corresponde a hidrocarburos. En ciudades grandes como Quito el 97\% de las emisiones de monóxido de carbono (CO) es aportado por las fuentes móviles, el 56\% de las emisiones de dióxido de azufre (SO2) tuvieron origen en las fuentes móviles y un $25.7 \%$ de las otras fuentes (fijas) corresponde a las centrales de generación termoeléctrica. El $80 \%$ de las emisiones de dióxido de nitrógeno (NO2) proceden de fuentes móviles, principalmente de buses pesados (42\%) y particulares livianos, taxis y camionetas (35\%) (Ministerio del Ambiente, 2008).

Otro factor contaminante es el ruido ambiental urbano provocado por la necesidad humana de trasladarse de un lugar a otro a causa de sus actividades productivas, generando el flujo vehicular libre y el flujo semáforo. A esto se suman otros elementos como la densidad poblacional, el uso de suelo, la construcción de edificaciones, la geometría urbana y el trazado vial.

La contaminación atmosférica es generada por material sedimentable que produce partículas PM10 y PM2.5 ${ }^{3}$, y por residuos o productos secundarios gaseosos, sólidos o líquidos, que ponen en peligro la salud del ser humano y de la biodiversidad, deteriora el patrimonio cultural, reduce la visibilidad y produce olores desagradables. Para el caso del presente estudio se realizó la medición de partículas PM 2.5.

Las partículas en suspensión provienen en parte de causas naturales como la erosión, los incendios forestales y las lluvias, entre otros. Estas patículas se sedimentan en el suelo según su composición y tamaño: las partículas cercanas a 10 micras de diámetro tienen un bajo poder de

\footnotetext{
${ }^{3}$ Las partículas entre 2,5 y 10 micras de diámetro se conocen como "gruesas". Las fuentes de las partículas gruesas son las operaciones de trituración o molienda, y el polvo levantado por los vehículos que circulen en las carreteras. (Consejería de Sanidad Región de Murcia, 2016)
} 
sedimentación, pero las que superan las 20 micras se depositan con facilidad y hay casos que superan las 300 micras de diámetro (Gobierno Vasco, 2006); además, los fenómenos meteorológicos transportan partículas de arena al paso de vehículos por vías cuya capa de rodadura es de tierra. Estos residuos se depositan en las viviendas, comercios, plantaciones, mobiliario urbano, y otros, siendo perjudiciales para la salud de la población. Cuando superan los límites de tolerancia ocasionan afecciones respiratorias principalmente a los grupos más vulnerables, constituidos por niños y ancianos (Carmona, 2012).

No existen estudios anteriores relacionados con el material particulado sedimentable en la ciudad de Pujilí, por este motivo la investigación constituye un aporte fundamental para iniciar y complementar la temática utilizando otros métodos que permitan generar nuevos aportes. Bajo esta consideración el objetivo de este estudio es obtener información preliminar de la concentración de material particulado en el área urbana de la ciudad, que justifique y promueva acciones por parte del GADM de Pujilí en relación a la calidad del aire, y a la vez aporte datos a proyectos de investigación sobre la movilidad urbana en ciudades intermedias de la Sierra Centro del Ecuador.

\section{Materiales y Métodos}

La investigación realizada es de tipo experimental y el estudio de campo se efectuó en el área urbana de la ciudad de Pujilí ubicada en la zona centro occidental de la provincia de Cotopaxi, a $10 \mathrm{~km}$ al oeste de la ciudad de Latacunga, en las coordenadas $78^{\circ} 41^{\prime} 44^{\prime \prime}$ W Y $00^{\circ} 5727^{\prime \prime} \mathrm{S}$, con un área de $8.85 \mathrm{~km}^{2}$ y a $2945 \mathrm{msnm}$. Su población urbana es de 10.064 habitantes (INEC, 2010), y de acuerdo a este peso poblacional y a su forma de distribución en el territorio está categorizada como ciudad intermedia tipo B.

Las variables físicas ambientales en las que fue realizado el trabajo de campo fueron: clima nublado, parcialmente nublado, soleado y parcialmente soleado. Temperatura mínima $21.3^{\circ} \mathrm{C}$ máxima $26.10^{\circ} \mathrm{C}$, entre $9 \mathrm{H} 00$ y $16 \mathrm{H} 00$, de acuerdo a los datos levantados en campo. La humedad relativa registra valores promedios en el rango de $88 \%$ a $96 \%$, mientras que la velocidad máxima promedio y la dirección del viento registrada es: al norte $12 \mathrm{~m} / \mathrm{s}$ y al noroeste $13 \mathrm{~m} / \mathrm{s}$ (B \& G consultores, 2015).

Para la medición de material particulado sedimentable se utiliza el método gravimétrico, mediante captación de partículas en envases abiertos, establecido por la norma vigente de calidad de aire del ambiente (MAE, 2013).

El proceso de preparación del equipo inició con la limpieza de envases colectores plásticos de 15 $\mathrm{cm}$ de diámetro, secado de los envases colectores en una estufa a $600^{\circ} \mathrm{C}$, pesado de los envases en balanza analítica calibrada, ubicación de los recipientes colectores en cada uno de los puntos 
establecidos a 1.2 metros sobre el nivel del suelo, en espacio abierto alejado de muros verticales, edificios y árboles.

El procedimiento de medición de material particulado sedimentable consistió en la codificación de los colectores de acuerdo a sus coordenadas de ubicación, inspección semanal de la muestra para garantizar su permanencia e integridad, recolección de los recipientes una vez transcurridos 30 días, secado de las muestras en una estufa a $600^{\circ} \mathrm{C}$ a fin de evaporar agua o impurezas y pesado final.

La selección de puntos para la toma de muestras fue realizada en base a criterios de densidad poblacional, densidad edificada, flujo vehicular y características de la capa de rodadura de las vías, a partir de ello fueron identificados seis sectores urbanos para la toma de muestras (ver Figura1).

La colocación de los puntos de muestreo fue realizada atendiendo a criterios de accesibilidad, seguridad, tránsito vehicular, consolidación urbana (según Tabla 1) en correspondencia con los objetivos de la investigación.

Tabla 1. Criterios de ubicación de las muestras

\begin{tabular}{|c|l|}
\hline Criterios & \multicolumn{1}{c|}{ Características } \\
\hline Accesibilidad & $\begin{array}{l}\text { Sitio de fácil acceso peatonal y vehicular que permita realizar las } \\
\text { revisiones periódicas del recipiente. }\end{array}$ \\
\hline Seguridad & Lugar que garantice la permanencia del recipiente. \\
\hline Tránsito vehicular & $\begin{array}{l}\text { Volumen de flujos vehiculares, importancia de la vía, tipo de capas } \\
\text { de rodadura de asfalto, adoquín y tierra; días y horario de actividad } \\
\text { laboral. }\end{array}$ \\
\hline $\begin{array}{c}\text { Consolidación } \\
\text { urbana }\end{array}$ & $\begin{array}{l}\text { Área con viviendas de baja, media y alta densidad, o cercana a } \\
\text { centros atractores de movilidad: equipamientos administrativos, } \\
\text { escolares, de salud, recreativos. }\end{array}$ \\
\hline
\end{tabular}

Los lugares específicos para el conteo de flujo vehicular fueron ubicados de acuerdo al mayor volumen de tránsito vehicular, la importancia de las vías, días de actividad laboral y por el tipo de capas de rodadura de asfalto, adoquín o tierra (ver Tabla 2). De acuerdo a estos criterios el conteo se realizó en la calle Av. Velasco Ibarra que pertenece a la red vial urbana principal de Pujilí, atraviesa a la ciudad de norte a sur y tiene mayor volumen de tráfico por ser una vía de conexión para trasladarse a los cantones Pangua y La Maná, y a sitios de importancia turística como la laguna del Quilotoa y la comunidad de Tigua.

Para contabilizar el flujo vehicular se llenaron tablas de acuerdo a categorías de tamaño y características de vehículos, en la siguiente clasificación: maquinaria agrícola, tracto camión, volqueta, camión, bus, buseta, camioneta, automóvil y motocicleta (INEN, 2012). Los sectores seleccionados fueron los mismos de la toma de material particulado, en días laborales y de $9 \mathrm{H} 00$ a $15 \mathrm{H} 00$. 


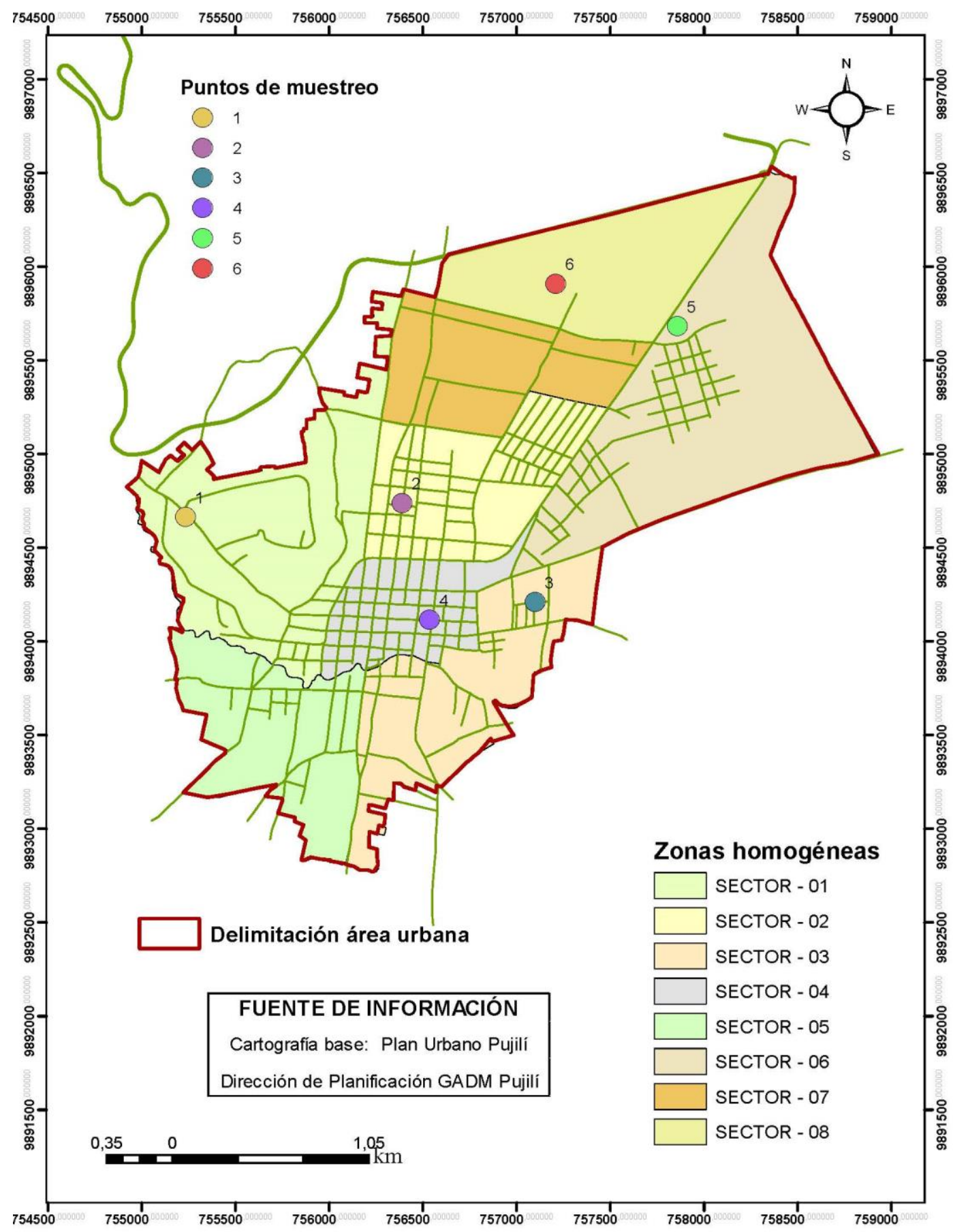

Figura 1. Mapa de ubicación de puntos de muestreo

Tabla 2. Datos de ubicación de los sitios de muestreo

\begin{tabular}{|c|c|c|c|c|c|c|}
\hline \multirow{2}{*}{ Punto } & \multirow{2}{*}{ Sector } & \multicolumn{2}{|c|}{ Coordenadas UTM } & Calle & Material de & vía \\
\cline { 3 - 4 } & 1 & 755231,65 & 9894664,86 & García Moreno & asfalto & Sinchaguasín \\
\hline 1 & 2 & 756387,59 & 9894739,23 & Luis Antonio Rivadeneira & asfalto & Simón Bolívar \\
\hline 2 & 3 & 757097,36 & 9894209,73 & $\begin{array}{c}\text { Raquel Abad y Rafael } \\
\text { Morales }\end{array}$ & adoquín & Calvario \\
\hline 3 & 4 & 756532,11 & 9894115,03 & $\begin{array}{c}\text { García Moreno y Atilino } \\
\text { Cajas }\end{array}$ & adoquín & Centro \\
\hline 4 & 7 & 757854,83 & 9895683,20 & Av. Velasco Ibarra & asfalto & Miraflores \\
\hline 5 & 8 & 757206,53 & 9895909,94 & C y Rafael Villacís & tierra & Bellavista \\
\hline 6 & & &
\end{tabular}




\section{Resultados}

En el punto de muestreo 6, se observa que la medición de material particulado sedimentable llega a $18.38 \mathrm{mg} / \mathrm{cm} 2$ en 30 días, es decir que supera el valor de la normativa vigente del anexo 4 del libro VI del Texto Unificado de la Legislación Ambiental Secundaria, que indica que para las partículas sedimentables, en un tiempo de exposición de 30 días, la concentración máxima permisible es de $1 \mathrm{mg} / \mathrm{cm} 2$. El punto 5 se acerca al límite máximo de lo que establece la norma. En la Tabla 3 y en la Figura 2 se presentan los resultados de material particulado sedimentable (PM 2.5), cuyo procedimiento de obtención se fundamenta en la citada norma ${ }^{4}$.

Tabla 3. Resultados del muestreo de material particulado sedimentable en los puntos de muestreo.

\begin{tabular}{|l|r|}
\hline \multicolumn{1}{|c|}{ Punto de muestreo } & \multicolumn{2}{c|}{$\begin{array}{c}\text { Material Particulado Sedimentable Acumulado } \\
(\mathbf{m g} / \mathbf{c m} \mathbf{2} \times \mathbf{3 0 d})\end{array}$} \\
\hline P1: Sinchaguasín & 0.11 \\
\hline P2: Simón Bolívar & 0.11 \\
\hline P3: Calvario & 0.24 \\
\hline P4: Centro & 0.08 \\
\hline P5: Miraflores & 0.74 \\
\hline P6: Bellavista & 18.38 \\
\hline
\end{tabular}

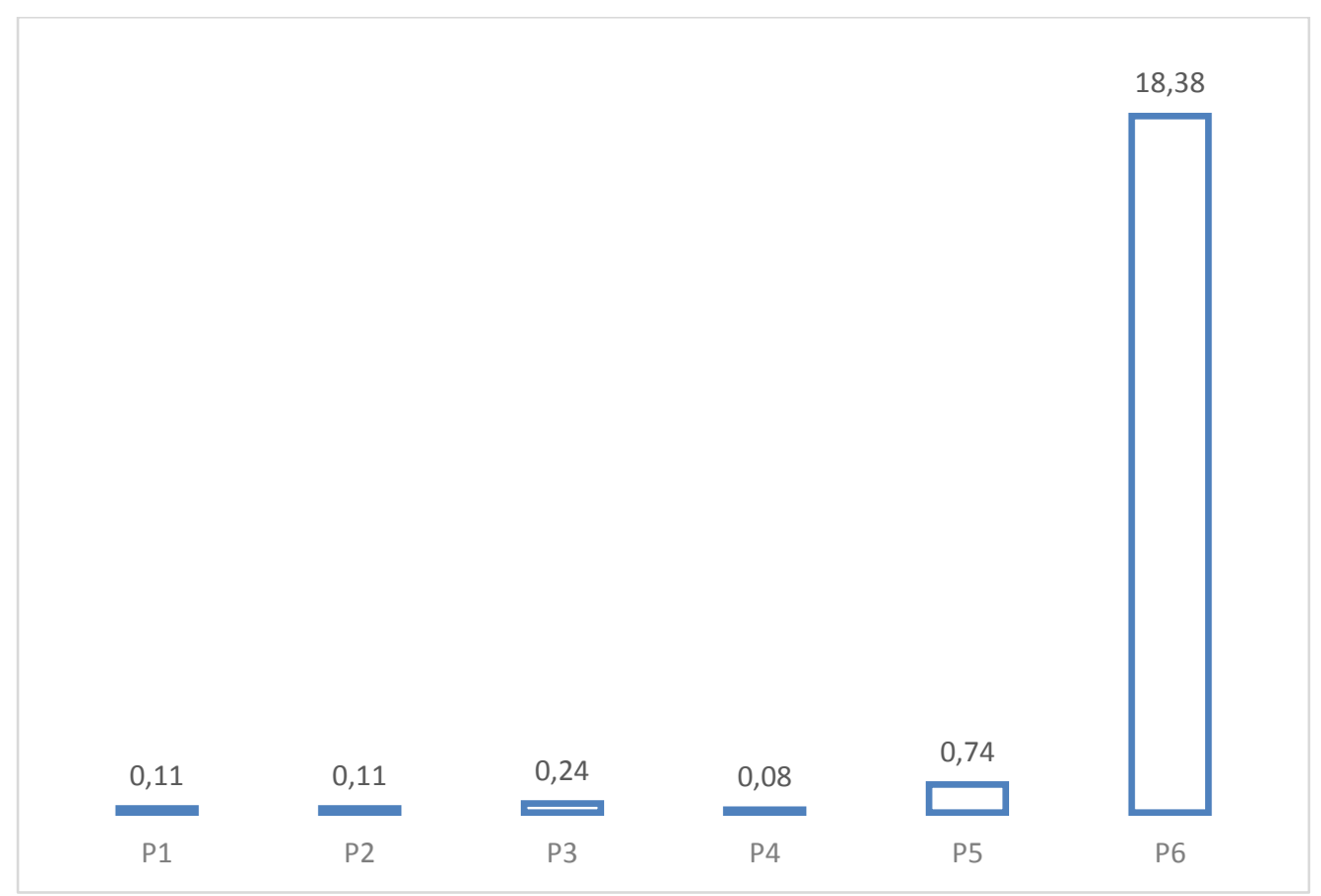

Figura 2. Resultados del muestreo de material particulado sedimentable

\footnotetext{
${ }^{4}$ Norma de calidad del aire ambiente libro VI anexo 4. Punto 4.1.4: De los métodos de medición de los contaminantes comunes del aire ambiente, Tabla 2
} 
Las mediciones observadas de concentraciones de contaminantes comunes del aire deberán corregirse de acuerdo a las condiciones de la localidad en que se efectúen dichas mediciones, para lo cual se utilizará el método gravimétrico, mediante captación de partículas en envases abiertos (Lodge, 1988).

De las mediciones observadas de concentraciones deben corregirse de acuerdo con la Ecuación 1, obteniendo los resultados que se exponen en la Tabla 4 y en la Figura 3, observándose que en la zona urbana de Pujilí, en el sector de Bellavista, existe mayor cantidad de polvo acumulado.

$$
C c=C o * \frac{760 \mathrm{mmHg}}{P b l m m H g} * \frac{\left(273+t^{\circ} \mathrm{C}\right)^{\circ} \mathrm{K}}{298^{\circ} \mathrm{K}},[1]
$$

Donde:

Cc: concentración corregida

Co: concentración observada

$\mathrm{Pbl}$ : presión atmosférica local, en milímetros de mercurio.

$t^{\circ} \mathrm{C}$ : temperatura local, en grados centígrados

Pbl: $540 \mathrm{mmHg}$

T으 : $18.2 \stackrel{\circ}{\circ} \mathrm{C}$

Tabla 4. Material Particulado PM2.5 Corregido en los diferentes puntos de muestreo

\begin{tabular}{|l|r|}
\hline \multicolumn{1}{|c|}{ Punto de muestreo } & \multicolumn{2}{c|}{$\begin{array}{c}\text { Material Particulado Sedimentable } \\
\text { Acumulado (mg/cm2x30d) }\end{array}$} \\
\hline P1: Sinchaguasín & 0.15 \\
\hline P2: Simón Bolívar & 0.15 \\
\hline P3: Calvario & 0.33 \\
\hline P4: Centro & 0.11 \\
\hline P5: Miraflores & 1.02 \\
\hline P6: Bellavista & 25.27 \\
\hline
\end{tabular}

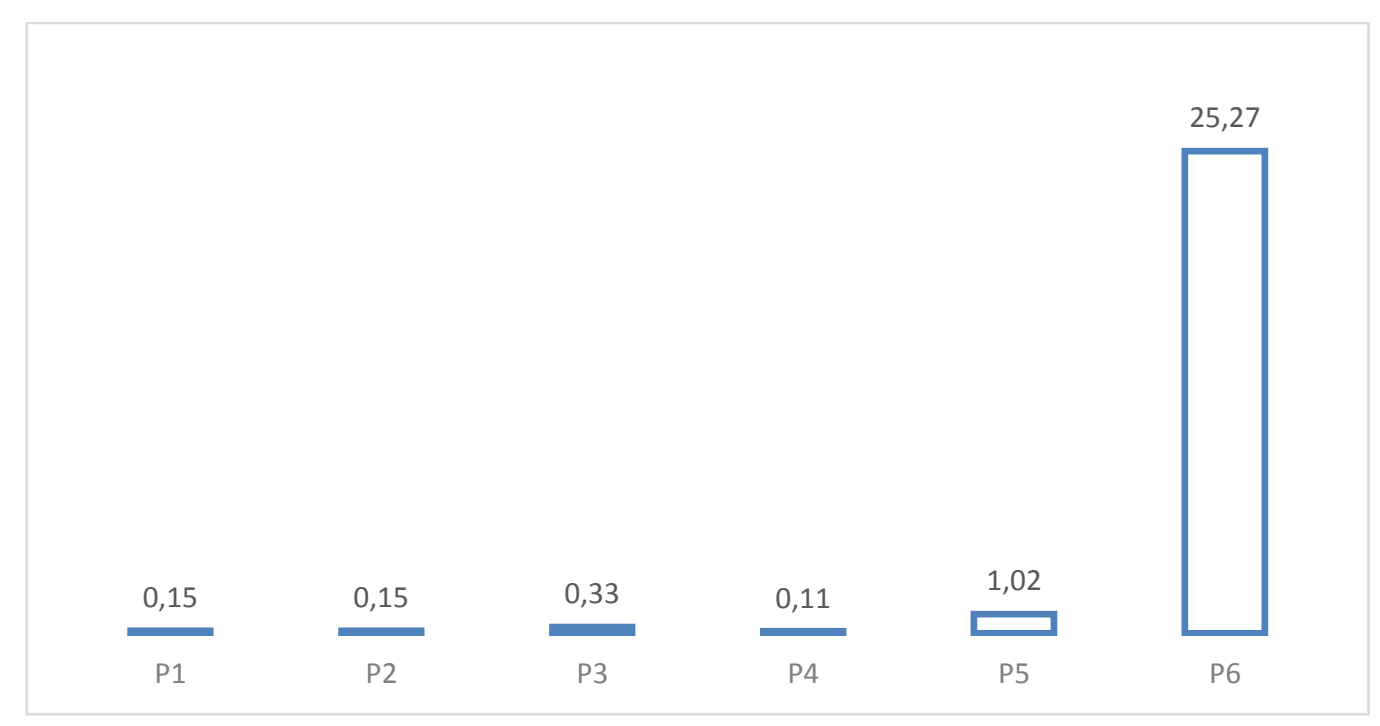

Figura 3. Material Particulado Sedimentable Acumulado en (mg/cm2×30d) 
La relación entre la tipología del parque automotor y el número de vehículos motorizados se observa en la Figura 4; en total se registraron 5865 unidades, donde los valores más altos, 3673 y 1774, corresponden a vehículos livianos y camionetas respectivamente. Con un número mucho menor se registran buses de transporte público: 258 (4,39\%) en total. Esto evidencia la preferencia de la población por el uso del vehículo privado, con el agravante de que el servicio de transporte público es limitado, situación que ha llevado a la población a utilizar camionetas para transportarse y llevar su carga.

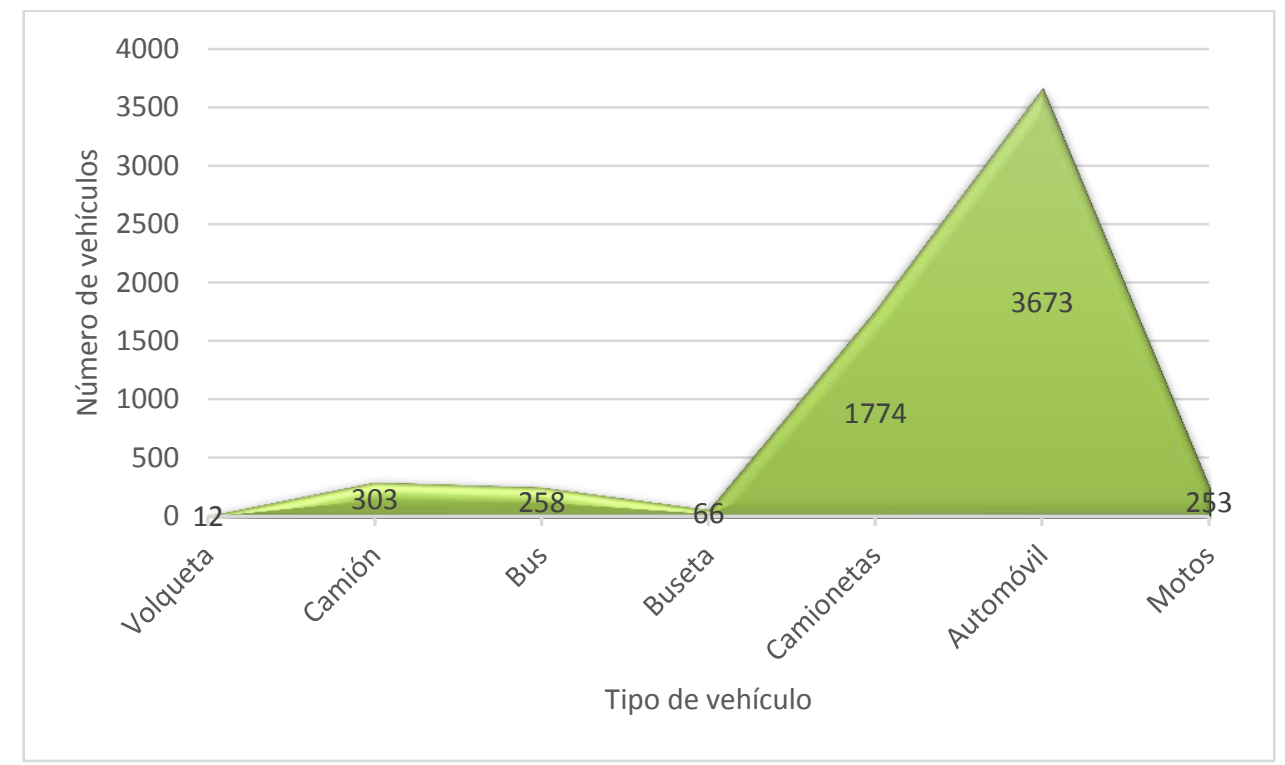

Figura 4. Fuentes móviles que incrementan la contaminación del aire

\subsection{CONTAMINACIÓN ATMOSFÉRICA Y AFECCION A LA SALUD}

De acuerdo a la información del Plan de Gobierno del GAD Municipal del Cantón Pujilí (20142019) se identifican dos subcentros de salud: San Gerardo y Juigua Yacubamba, regentados por el Ministerio de Salud, así como cinco dispensarios del Seguro Social Campesino en los que las enfermedades más comunes que afectan a la población son infecciones respiratorias agudas. Al momento se está estudiando la incidencia que tiene el material particulado y la tasa de morbilidad por IRA (infecciones respiratorias agudas) en la zona de estudio.

\section{Discusión}

La investigación evidencia la existencia de la problemática en ciudades pequeñas en desarrollo, por este motivo los gobiernos locales deben iniciar el levantamiento de diagnósticos que tomen en cuenta los impactos ambientales generados por los procesos de urbanización, en este caso para las emisiones contaminantes, tomando en cuenta que el medio ambiente como receptor de fluentes (emisiones, vertidos y residuos no deseados), no deberá sobrepasar su capacidad de asimilación (Conesa \& Conesa, 2013). 
La actualidad y utilidad técnica de la investigación causa expectativa a nivel de ciudades mayores o ciudades intermedias tipo A en la región andina, cuyas poblaciones sobrepasan los 20.000 habitantes, pero la verdadera problemática ambiental provocada por la movilidad en las ciudades intermedias tipo B ubicadas en la sierra centro del país aún no emerge, sobre todo si estas localidades urbanas ubicadas por encima de los 2.000 m.s.n.m, sirven de nexo o de interconectividad entre centros urbanos y los centros de producción agrícola y con igual derecho a la calidad de vida con características ambientales óptimas.

Se demuestra que la esencia de los problemas ambientales reside en el comportamiento de los agentes socioeconómicos, productores-consumidores de bienes y servicios (Gómez, 2007); la ausencia de una planificación adecuada en cuanto a movilidad lleva a que los actores asimilen hábitos que tienden a satisfacer necesidades individuales en detrimento del ambiente, por el incremento del vehículo particular

El estudio realizado aporta con información base para la elaboración de modelos participativos de gestión urbana, así como para el despliegue de estrategias de rehabilitación, mejoramiento y mantenimiento de la infraestructura vial, respetando el medio ambiente y áreas de riesgo socio natural; a la vez promueve acciones iniciales de regulación, control y monitoreo en relación a la calidad del aire que requiere gestionar el GADM de Pujilí.

Debido a que las mediciones de concentraciones de contaminantes comunes del aire deberán corregirse de acuerdo a las condiciones de la localidad en que se efectúen dichas mediciones y a una posible pérdida de masa durante la manipulación de la muestra, a futuro se requiere realizar mediciones periódicas utilizando el método gravimétrico en otros sectores críticos de la ciudad de Pujilí.

Se debe incentivar estudios e investigaciones que permitan aportar con información actualizada para una mejor precisión de las fuentes contaminantes y que el Gobierno Cantonal pueda establecer una normativa para regular la contaminación proveniente de las distintas fuentes y aumentar el control de las emisiones producidas por el parque automotor de Pujilí.

\section{Conclusiones}

Como mencionan las normas de calidad de aire a nivel internacional (IDEAM, 2004), indica que se considera a las partículas sedimentables como contaminantes no convencionales, cuyo valor máximo permisible de sedimentación es $1 \mathrm{mg} / \mathrm{cm} 2$ en un mes, en los países de Argentina y Ecuador. En este caso, se detectó en Bellavista la mayor cantidad de material particulado que es superior al que indica el Convenio de Asociación; así mismo se atribuye como una de las principales fuentes de generación de contaminación el transporte del sector, debido a que son áreas en las que se concentra tanto el transporte liviano como pesado. 
Los datos recopilados demuestran que Bellavista (P6) es el sector donde existe mayor cantidad de material particulado sedimentable causado por tres tipos de fuentes vehiculares recurrentes como son las camionetas, motos o automóviles, utilizados para carga y desplazamiento poblacional a través de vías lastradas o de tierra que se articulan a las vías colectoras y recogen el tráfico de poblaciones menores o zonas de tipo rural generalmente de sectores productivos agrícolas. En contraste existe únicamente un $4,39 \%$ de buses para transporte público generalmente utilizados para movilización en vías asfaltadas.

Las emisiones de material particulado en trayectos no pavimentados son producidas por la pulverización del material superficial de suelo causado por la velocidad de circulación de los vehículos; a esto se suma la acción del viento y la presencia de lotes baldíos que contribuyen a incrementar la presencia de material particulado en el aire, lo que expone a las personas a dolencias en las vías respiratorias por el material inhalado.

\section{Bibliografía}

B \& G consultores. (2015). PDOT GADM Pujilí. Pujilí.

Carmona, J. A. (enero de 2012). Modelo de valoración ambiental del impacto de la contaminación Atmosférica por fuentes móviles en el Municipio de Pereira.

Conesa, V., \& Conesa, L. (2013). Guía metodológica para la evaluación del impacto ambiental. Madrid: Mundi-Prensa.

Consejería de Sanidad Región de Murcia. (2016). Portal sanitario de la Región de Murcia. Obtenido de http://www.murciasalud.es/pagina.php?id=244308\&idsec=1573\#

GADP de Cotopaxi. (2015). Actualización del Plan de Desarrollo y Ordenamiento Territorial de Cotopaxi 2025.

Gobierno Vasco. (2006). Estudio de los niveles de PM10 y PM2.5 en una área urbana con influencia industrial siderometalurgia. Basauri, Vizcaya: Ingurumena.net.

Gómez, D. (2007). Evaluación ambiental estratégica . Madrid: Mundi-Prensa.

IDEAM. (2004). Convenio de Asociación №038/04. Bogotá: Subdirección de estudios ambientales.

INAMHI. (2015). Servicio meteorológico. Obtenido de http://www.serviciometeorologico.gob.ec/

INEC. (2010). Ecuador en cifras. Obtenido de http://www.ecuadorencifras.gob.ec/

INEN. (2012). Norma técnica ecuatoriana Clasificación Vehicular. Quito.

Instituto Nacional de Meteorología e hidrología INAMHI. (2015). Anuario Meteorológico No. 522012. Quito: Dirección Ejecutiva del INAMHI. 
Lodge, J. (1988). Methods of air sampling and analysis. Washington: CRC Press.

MAE. (2013). Texto unificado de legislacion ambiental secundario. Obtenido de http://www.ambiente.gob.ec/biblioteca/

Ministerio del Ambiente. (2008). Informe sobre el estado del Medio Ambiente. Quito: Ministerio del Ambiente.

Organización Panamericana de la Salud. (2005). Evaluación de los Efectos de la Contaminación del Aire en la Salud de América Latina y el Caribe. Washington, DC: Organización Panamericana de la Salud. 